\title{
ON THE MÖBIUS FUNCTION
}

\author{
HELMUT MAIER
}

\begin{abstract}
We investigate incomplete convolutions of the Möbius function of the form $\sum_{d \mid n ; d \leq z} \mu(d)$. It is shown that for almost all integers $n$ one can find $z$ for which this sum is large.
\end{abstract}

1. Introduction. The function $M(n)=\sup _{z \leq n}\left|\sum_{d \mid n ; d \leq z} \mu(d)\right|$ has been studied in various papers $[\mathbf{1}, \mathbf{2}, \mathbf{5}]$. Erdös and Katai $[\overline{\mathbf{2}}]$ proved that

$$
M(n) \leq A^{\omega(n)}
$$

if $A>\sqrt{2}$.

(We use (p.p.) to indicate that a property holds on a sequence of asymptotic density 1.)

This result was improved by Hall [5], who showed that $A>3 / e$ is sufficient.

A recent result of $G$. Tenenbaum and the author $[9]$ implies almost immediately

THEOREM 1 .

$$
M(n) \leq \psi(n) \log \log n,
$$

where $\psi(n)$ is any function tending to $\infty$.

PROOF. Let $p_{1}(n)$ be the smallest prime factor of $n$. Then $\mu(d)+\mu\left(p_{1}(n) d\right)=0$ for all $d \not \equiv 0 \bmod p_{1}(n)$. Therefore

$$
M(n) \leq \sup _{z \leq n} \sum_{\substack{z<d \leq z p_{1}(n) \\ d \mid n}} 1 \mid .
$$

In $[9]$ it is shown that

$$
\Delta(n) \leq \psi(n) \log \log n,
$$

where $\Delta$ is Hooley's function [7], defined by

$$
\Delta(n)=\sup _{z \leq n} \sum_{z \leq d<e z} 1 .
$$

It follows by sieve methods that

$$
p_{1}(n) \leq \psi(n)
$$

Received by the editors December 19, 1985.

1980 Mathematics Subject Classification (1985 Revision). Primary 11K65; Secondary $11 B 05$.

Research supported by an NSF Grant. 
Thus

$$
\begin{gathered}
M(n) \leq \sup _{z \leq n}\left|\sum_{\substack{z<d \leq z p_{1}(n) \\
d \mid n}} 1\right| \leq \Delta(n) \log \psi(n) \\
\leq \psi(n) \log \psi(n) \log \log n .
\end{gathered}
$$

Since $\psi(n)$ was arbitrary Theorem 1 follows.

In [1 and 2] the question for a lower bound for $M(n)$ was raised. The purpose of this paper is to establish such a lower bound.

We will prove

THEOREM 2. Let

$$
\gamma<-\frac{\log 2}{\log \left(1-(\log 3)^{-1}\right)}=0.28754 \ldots
$$

Then

$$
M(n) \geq(\log \log n)^{\gamma} .
$$

Many of the techniques applied will be very similar to those applied in [8], where the same lower bound was obtained for $\Delta(n)$. However we need also some new devices which bear resemblance to those used in [9].

2. Notations and preliminary lemmas. We fix a function $v(x)$, to be specified later, with $v(x) \rightarrow \infty(x \rightarrow \infty)$ and also a constant $\rho>1$.

Based on these two parameters we define $r_{k}=\rho^{k} v(x)$ and $r_{k, l}=\rho^{k} v(x)+l$ where $k$ and $l$ are any nonnegative integers. For any positive integer $n \leq x$ and a real number $z>0$ we set

$$
n_{z}=\prod_{\substack{\log \log p<z \\ p \mid n}} p, \quad n_{z}^{*}=\prod_{\substack{\log \log p<z \\ p^{\nu p} \| n}} p^{\nu p} .
$$

We use $n_{(k)}$ for $n_{r_{k}} ; n_{(k, l)}$ for $n_{r_{k, l}} ; n_{(k)}^{*}$ for $n_{r_{k}}^{*} ;$ and $n_{(k, l)}^{*}$ for $n_{r_{k, l}}^{*}$. We define

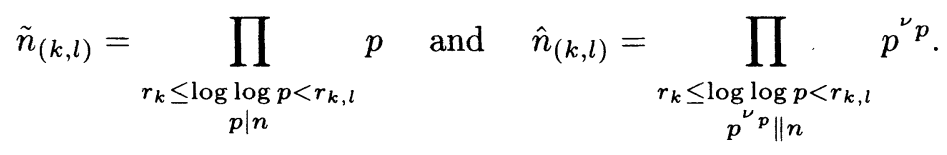

Assume that

$$
n=n_{(k)}^{*} p_{1}^{(k)^{\nu_{1}}} \cdots p_{t(n)}^{(k)^{\nu_{t(n)}}}, \quad p_{1}^{(k)}<\cdots<p_{t(n)}^{(k)}
$$

Then we set

$$
\begin{array}{ll}
\tilde{n}_{(k)}^{(s)}=\prod_{t \leq s} p_{t}^{(k)}, & \hat{n}_{(k)}^{(s)}=\prod_{t \leq s} p_{t}^{(k)^{\nu t}} \\
n_{(k)}^{(s)}=n_{(k)} \tilde{n}_{(k)}^{(s)}, & n_{(k)}^{*(s)}=n_{(k)}^{*} \hat{n}_{(k)}^{(s)} .
\end{array}
$$


For any quadruplet $(n, k, l, \eta)$, where $n \leq x, k, l$ nonnegative integers, and $\eta>0$ we denote by $\mu(n, k, l, \eta)$ the Lebesgue measure of the set

$$
\mathcal{E}(n, k, l, \eta)=\bigcup_{\substack{d d^{\prime} \mid \tilde{n}_{(k, l)} \\ \mu\left(d d^{\prime}\right)=1}}\left(\log \frac{d^{\prime}}{d}\right)+[-\eta, \eta] .
$$

We now proceed with some auxiliary lemmas. They all are either identical or rather similar to the lemmas applied in [8].

LEMMA 1. Let $f$ be a nonnegative multiplicative function such that for all primes $p$

$$
0 \leq f\left(p^{\nu}\right) \leq \lambda_{1} \lambda_{2}^{\nu} \quad(\nu=1,2, \ldots),
$$

where $0<\lambda_{1}, 0<\lambda_{2}<2$. Then for $x \geq 1$

$$
\sum_{n \leq x} f(n) \ll_{\lambda_{1}, \lambda_{2}} x \prod_{p \leq x}\left(1-p^{-1}\right) \sum_{\nu=0}^{\infty} f\left(p^{\nu}\right) p^{-\nu} .
$$

This is a weakening of a theorem of Halberstam and Richert [4] generalizing a result of Hall.

LEMMA 2. For $2 \leq u \leq v \leq x$, we have

$$
\operatorname{card}\left\{n \leq x: \prod_{p \leq u, p^{\nu} \| n} p^{\nu} \geq v\right\} \ll x \exp \left(-c_{0} \frac{\log v}{\log u}\right)
$$

where $c_{0}>0$ is an absolute constant.

This is established in $[\boldsymbol{3}]$ and, in a stronger version, in $[\mathbf{1 0}]$.

LEMMA 3. Let $u(x)$ be any function tending to $\infty$ such that $u(x) \leq \log \log x$. Let $\delta_{0}>0$ be a fixed constant. Then for each $r$ with $u(x) \leq r \leq \log \log x$ we have uniformly in $s, u(x) \leq s \leq r$,

$$
\left|\omega\left(n_{r} / n_{r-s}\right)-s\right| \leq \delta_{0} s
$$

for all $n \leq x$ except a set of cardinality $\ll \delta_{0} x \exp \left(-c\left(\delta_{0}\right) u(x)\right)$, where $c\left(\delta_{0}\right)>0$ depends only on $\delta_{0}$.

PROOF. We first estimate the number of integers $n \leq x$ for which $\omega\left(n_{r} / n_{r-s}\right) \leq$ $s\left(1-\delta_{0}\right)$ for any integer $s$. By Lemma 1 this number does not exceed

$$
\sum_{u(x) \leq s \leq r} \sum_{n \leq x} \alpha^{\omega\left(n_{\digamma} / n_{r-s}\right)-\alpha s} \ll_{\alpha} x \sum_{s \geq u(x)} e^{-Q(\alpha) s},
$$

where $\alpha=1-\delta_{0}, Q(\alpha)=\alpha \log \alpha-\alpha+1>0$. The number of integers $n \leq x$ for which $\omega\left(n_{r} / n_{r-s}\right) \geq s\left(1+\delta_{0}\right)$ for any $s$ does not exceed

$$
\sum_{u(x) \leq s \leq r} \sum_{n \leq x} \beta^{\omega\left(n_{r} / n_{r-s}\right)-\beta s} \ll_{\beta} x \sum_{s \geq u(x)} e^{-Q(\beta) s},
$$

where $\beta=1+\delta_{0}, Q(\beta)>0$. 
LEMMA 4. Let $w(x)$ be any function defined for $x \geq 1$ such that $w(x)>1$, $w(x) \rightarrow \infty$ for $x \rightarrow \infty$, and $v(x) \geq w(x)$. Moreover, we asume that

$$
l \geq \rho^{k} v(x)(\log 3-1)^{-1}\left(1+\delta_{1}\right)
$$

$$
\text { for some } \delta_{1}>0, r_{k, l} \leq \log \log x \text { and } 1 \geq \eta \geq 1 / r_{k} \text {. }
$$

Then there exists $c_{1}=c_{1}\left(\delta_{1}\right)>0$ such that $\mu(n, k, l, \eta) \geq \exp \left(r_{k, l}\right) w(x)^{-2}$ for all $n \leq x$ except a set of cardinality $\ll_{\delta_{1}} x w(x)^{-c_{1}}$.

Proof. Set

$$
F(z)=F(k, l, z)=\sum_{\substack{d d^{\prime} \mid \tilde{n}_{(k, l)} ; \log \left(d^{\prime} / d\right) \leq z \\ \mu\left(d d^{\prime}\right)=1}} 1 .
$$

The $\mu(n, k, l, \eta)$ is the measure of the set of those $z$ for which $F(z+\eta)-F(z-\eta) \neq 0$. We introduce the exponential sum

$$
S_{k, l}(\theta, n)=\sum_{\substack{d d^{\prime} \mid \tilde{n}_{(k, l)} \\ \mu\left(d d^{\prime}\right)=1}}\left(d^{\prime} / d\right)^{i \theta} .
$$

We have

$$
\begin{aligned}
F(z+\eta)-F(z-\eta) & \leq 2 \int_{-\infty}^{\infty}\left(\frac{\sin ((u-z) / 2 \eta)}{(u-z) / 2 \eta}\right)^{2} d F(u) \\
& =2 \eta \int_{-1 / \eta}^{1 / \eta} e^{i \theta z}(1-|\theta \eta|) S_{k, l}(\theta, n) d \theta
\end{aligned}
$$

by Parseval's formula.

A second application of this formula implies

$$
\int_{-\infty}^{\infty}(F(z+\eta)-F(z-\eta))^{2} d z \leq 8 \pi \eta^{2} \int_{-1 / \eta}^{1 / \eta}(1-|\theta \eta|)^{2} S_{k, l}(\theta, n)^{2} d \theta
$$

This together with

$$
\begin{aligned}
\left(2 \eta 3^{\omega\left(\tilde{n}_{(k, l)}\right)-1}\right)^{2} & \leq\left(\int_{-\infty}^{\infty}(F(z+\eta)-F(z-\eta)) d z\right)^{2} \\
& \leq \mu(n, k, l, \eta) \int_{-\infty}^{\infty}(F(z+\eta)-F(z-\eta))^{2} d z
\end{aligned}
$$

gives

$$
\mu(n, k, l, \eta) \geq 3^{2 \omega\left(\tilde{n}_{(k, l)}\right)-2}\left(2 \pi \int_{-1 / \eta}^{1 / \eta} S_{k, l}(\theta, n)^{2} d \theta\right)^{-1} .
$$

Thus to establish Lemma 4 it suffices to prove

$$
\int_{-1 / \eta}^{1 / \eta} S_{k, l}(\theta, n)^{2} d \theta \leq 3^{2 \omega\left(\tilde{n}_{(k, l)}\right)-2} e^{-r_{k, l}} w(x)^{2}(2 \pi)^{-1}
$$

for all $n \leq x$ except a set of cardinality $\ll_{\delta_{1}} x w(x)^{-c_{1}}$, where $c_{1}=c_{1}\left(\delta_{1}\right)$ is a suitable constant. For this purpose we decompose

$$
S_{k, l}(\theta, n)=\frac{1}{2}\left(S_{k, l}^{(1)}(\theta, n)+S_{k, l}^{(2)}(\theta, n)\right)
$$


where

$$
\begin{aligned}
S_{k, l}^{(1)}(\theta, n) & =\sum_{d d^{\prime} \mid \tilde{n}_{(k, l)}}\left(d^{\prime} / d\right)^{i \theta}=\prod_{p \mid \tilde{n}_{(k, l)}}(1+2 \cos (\theta \log p)), \\
S_{k, l}^{(2)}(\theta, n) & =\sum_{d d^{\prime} \mid \tilde{n}_{(k, l)}} \mu\left(d d^{\prime}\right)\left(d^{\prime} / d\right)^{i \theta}=\prod_{p \mid \tilde{n}_{(k, l)}}(1-2 \cos (\theta \log p)) .
\end{aligned}
$$

Since $S_{k, l}^{2} \leq \frac{1}{2}\left(S_{k, l}^{(1) 2}+S_{k, l}^{(2) 2}\right)$ it is sufficient to show that

$$
\int_{-1 / \eta}^{1 / \eta} S_{k, l}^{(i)}(\theta, n)^{2} d \theta \leq(2 \pi)^{-1} 3^{2 \omega\left(\tilde{n}_{(k, l)}\right)-2} e^{-r_{k, l}} w(x)^{2}
$$

for all $n \leq x$ except a set of cardinality

$$
\ll \delta_{1} x w(x)^{-c_{1}} \quad(i=1,2) .
$$

We show this only for $i=1$, the case $i=2$ being analogous.

For the range $|\theta| \leq \exp \left(-r_{k, l}\right) w(x)$ we take the trivial estimate

$$
\left|S_{k, l}^{(1)}(\theta, n)\right| \leq 3^{\omega\left(\tilde{n}_{(k, l)}\right)}
$$

and obtain

$$
\int_{|\theta| \leq \exp \left(-r_{k, l}\right) w(x)} S_{k, l}^{(1)}(\theta, n)^{2} d \theta \leq 2 \cdot 3^{2 \omega\left(\tilde{n}_{(k, l)}\right)} \exp \left(-r_{k, l}\right) w(x) .
$$

For the estimate of the contribution from the remaining range we introduce

$$
f_{\theta}(n)=S_{k, l}^{(1)}(\theta, n)^{2} z^{\omega\left(\tilde{n}_{(k, l)}\right)} y^{\omega_{\theta}\left(\tilde{n}_{(k, l)}\right)}
$$

with

$$
\omega_{\theta}(r)=\sum_{\substack{\log p \leq 1 /|\theta| \\ p \mid r}} 1
$$

and estimate $\sum_{n \leq x} f_{\theta}(n)$ by Lemma 1 .

We have $f_{\theta}(n)=\prod_{p \mid n} f(p)$, where

$$
f_{\theta}(p)= \begin{cases}(1+2 \cos (\theta \log p))^{2} y z, & \text { if } \exp \left(r_{k}\right) \leq \log p \leq \theta^{-1} \\ (1+2 \cos (\theta \log p))^{2} z, & \text { if } \theta^{-1}<\log p<\exp \left(r_{k, l}\right) \\ 1, & \text { otherwise }\end{cases}
$$

in the range $\exp \left(-r_{k, l}\right) w(x) \leq \theta \leq \exp \left(-r_{k}\right)$ and

$$
f_{\theta}(p)= \begin{cases}(1+2 \cos (\theta \log p))^{2} z, & \text { if } r_{k} \leq \log \log p<r_{k, l}, \\ 1, & \text { otherwise }\end{cases}
$$

in the range $\theta>\exp \left(-r_{k}\right)$.

We obtain for the first range

$$
\begin{aligned}
\sum_{n \leq x} f_{\theta}(n) \ll x \exp ( & \sum_{\exp \left(r_{k}\right) \leq \log p \leq \theta^{-1}} \frac{9 y z-1}{p} \\
& \left.+\sum_{\theta^{-1}<\log p<\exp \left(r_{k, l}\right)} \frac{z(1+2 \cos (\theta \log p))^{2}-1}{p}\right) \\
\ll x \exp \left\{(9 y z-1)\left(\log ^{+}\left(|\theta|^{-1}\right)-r_{k}+1\right)\right. & \left.+(3 z-1)\left(r_{k, l}-\log ^{+}\left(|\theta|^{-1}\right)\right)+O(z)\right\}
\end{aligned}
$$


the second sum over $p$ being estimated, using the prime number theorem as explained in [6, Lemma 4].

For the second range we obtain

$$
\begin{aligned}
\sum_{n \leq x} f_{\theta}(n) & \ll x \exp \left(\sum_{r_{k} \leq \log \log p<r_{k, l}} \frac{z(1+2 \cos (\theta \log p))^{2}-1}{p}\right) \\
& \ll x \exp \left\{(3 z-1) l+O_{z}(\log \log (3+|\theta|))\right\} .
\end{aligned}
$$

Now we choose $y=\frac{1}{3}, z=\frac{1}{3}$ and we obtain

$$
\sum_{n \leq x} f_{\theta}(n) \ll\left\{\begin{array}{l}
x, \quad \text { if } \exp \left(-r_{k, l}\right) w(x) \leq|\theta| \leq \exp \left(-r_{k}\right), \\
x(\log (3+|\theta|))^{c_{2}}, \quad \text { if } \exp \left(-r_{k}\right)<|\theta| \leq 1 / \eta,
\end{array}\right.
$$

where $c_{2}>0$ is an absolute constant.

To get estimates for $S_{k, l}^{(1)}(\theta, n)$ itself we need estimates for $\omega\left(\tilde{n}_{(k, l)}\right)$ and $\omega_{\theta}\left(\tilde{n}_{(k, l)}\right)$.

We set $\delta_{2}=\frac{1}{2}\left(1-(\log 3)^{-1}\right)$ and obtain by

LEMMA 3. $\omega\left(\tilde{n}_{(k, l)}\right)-\omega_{\theta}\left(\tilde{n}_{(k, l)}\right) \geq\left(1-\delta_{2}\right)\left(r_{k, l}-\log \left(|\theta|^{-1}\right)\right)$ in the range $\exp \left(-r_{k, l}\right) w(x)<|\theta| \leq \exp \left(-r_{k}\right)$ for all $n \leq x$ except a set of cardinality $\ll x \exp \left(-c_{1} \log w(x)\right)$ for an appropriate $c_{1}=c_{1}\left(\delta_{1}\right)>0$.

Together with (2.3) this yields

$$
\sum_{n \leq x}^{\prime} S_{k, l}^{(1)}(\theta, n)^{2} 3^{-2 \omega\left(\tilde{n}_{k, l}\right)} \ll x 3^{-\left(1-\delta_{2}\right)\left(r_{k, l}-\log \left(|\theta|^{-1}\right)\right)}
$$

for the range $\exp \left(-r_{k, l}\right) w(x)<|\theta| \leq \exp \left(-r_{k}\right)$, where the sum $\sum^{\prime}$ is extended over all $n \leq x$ except a set of cardinality $\ll x w(x)^{-c_{1}}$. Thus

$$
\begin{aligned}
\sum_{n \leq x}^{\prime} 3^{-2 \omega\left(\tilde{n}_{(k, l)}\right)} \int_{\exp \left(-r_{k, l}\right) w(x)<|\theta| \leq \exp \left(-r_{k}\right)} S_{k, l}^{(1)}(\theta, n)^{2} d \theta \\
\ll x \exp \left(-r_{k, l}\right) w(x)^{-\left(1-\delta_{2}\right) \log 3+1} .
\end{aligned}
$$

For the estimate of the integral over the second range $\exp \left(-r_{k}\right)<|\theta| \leq 1 / \eta$ we observe that because of $l \geq r_{k}(\log 3-1)^{-1}\left(1+\delta_{1}\right)$ we can find $\delta_{3}=\delta_{3}\left(\delta_{1}\right)>0$ such that

$$
l\left(\left(1-\delta_{3}\right) \log 3-1\right) \geq r_{k}\left(1+\delta_{3}\right) .
$$

$\left|\omega\left(\tilde{n}_{(k, l)}\right)-l\right| \leq \delta_{3} l$ for all $n \leq x$ except a set of cardinality $\ll_{\delta_{1}} x \exp \left(-c_{3}\left(\delta_{1}\right) w(x)\right)$, where $c_{3}>0$ depends only on $\delta_{1}$. Thus

$$
\begin{gathered}
\sum_{n \leq x}^{\prime}\left(\int_{\exp \left(-r_{k}\right)<|\theta| \leq 1 / \eta} S_{(k, l)}^{(1)}(\theta, n)^{2} d \theta\right) 3^{-2 \omega\left(\tilde{n}_{(k, l)}\right)} \\
\ll \frac{x}{\eta} 3^{-\left(1-\delta_{3}\right) l}\left(\log \left(3+\frac{1}{\eta}\right)\right)^{c_{2}}
\end{gathered}
$$

where $\sum^{\prime}$ means that the sum is extended over all $n \leq x$ except a set of cardinality $\ll \delta_{1} x \exp \left(-c_{3}\left(\delta_{1}\right) w(x)\right)$. But $3^{-\left(1-\delta_{3}\right) l} \ll \exp \left(-r_{k, l}\right) \exp \left(-\delta_{3} r_{k}\right)$. Now $(2.2),(2.4)$, (2.5) give that for $x \geq x_{0}$

$$
\sum_{n \leq x}^{\prime}\left(\int_{|\theta| \leq 1 / \eta} S_{(k, l)}^{(1)}(\theta, n)^{2} d \theta\right) 3^{-2 \omega\left(\tilde{n}_{(k, l)}\right)} \leq x e^{-r_{k, l}} w(x)^{2}
$$


where $\sum^{\prime}$ is extended over all $n \leq x$ except a set of cardinality $\ll_{\delta_{1}} x w(x)^{-c_{1}}$. This proves (2.1) and thus finishes the proof of Lemma 4.

3. Proof of Theorem 2. Given now

$$
\gamma<-\frac{\log 2}{\log \left(1-(\log 3)^{-1}\right)}
$$

then we fix $\varepsilon_{1}>0$ such that

$$
\left(1-10 \varepsilon_{1}\right) \frac{\log 2}{\gamma}>-\log \left(1-(\log 3)^{-1}\right) .
$$

Then we set

$$
\begin{aligned}
& \rho=\min \left(\exp \left(\left(1-8 \varepsilon_{1}\right) \frac{\log 2}{\gamma}\right), \frac{\log 3}{\log 3-1}+\frac{1}{2} \frac{1-\log 2}{\log 3-1}\right), \\
& v(x)=(\log \log x)^{6 \varepsilon_{1}}, \quad w(x)=(\log \log x)^{\varepsilon_{1}}, \\
& K=\left[\frac{1+\varepsilon_{1}}{\log 2} \gamma \log \log \log x\right] .
\end{aligned}
$$

These choices imply that $\rho^{K} v(x) \leq(\log \log x)^{1-\varepsilon_{1}}$ and $2^{K}>2(\log \log x)^{\gamma}$.

In the following considerations we always assume that $x$ is sufficiently large: $x \geq x_{0}(\gamma)$. We are now asking for blocks of divisors $d_{1}<d_{2}<\cdots<d_{s}$ such that $\mu\left(d_{1}\right)=\mu\left(d_{2}\right)=\cdots=\mu\left(d_{s}\right) \neq 0$, which are not interrupted by other divisors.

To make our demands more precise we introduce the two sequences:

$$
\xi_{k}=\frac{1}{100} \sum_{l=1}^{k} \frac{1}{l^{2}} \quad \text { and } \quad \zeta_{k}=\log 2-\frac{1}{100} \sum_{l=1}^{k} \frac{1}{l^{2}}, \quad k \geq 0 .
$$

Later we will still need

$$
\eta_{k}=1 / 100 k^{2} \text {. }
$$

We now introduce the property $B(k)$. We say that an integer $n \leq x$ has property $(B(k))$, if the following is true:

There are $2^{k}$ divisors of $n_{(k)}$ having the following property $(P(k))$.

$$
\begin{gathered}
d_{1}<\cdots<d_{2 k}, \quad \mu\left(d_{1}\right)=\cdots=\mu\left(d_{2 k}\right) \neq 0 \\
\left|\log d_{2 k}-\log d_{1}\right| \leq \xi_{k} \quad \text { and } \quad d \mid n, \mu(d) \neq 0, \\
d \notin\left\{d_{1}, \ldots, d_{2 k}\right\} \Rightarrow \log d<\log d_{1}-\varsigma_{k} \text { or } \log d>\log d_{2}+\varsigma_{k} .
\end{gathered}
$$

We will prove by induction in $k$ for $0 \leq k \leq K$ the statement $S(k)$ :

All integers $n \leq x$ have property $(B(k))$ except those of a set of cardinality $\leq c_{4}(\gamma) x w(x)^{-c_{5}(\gamma)}(k+1)$. If $k=K$ this means that all integers $n \leq x$ except a set of cardinality $\leq c_{4}(\gamma) x w(x)^{-c_{5}(\gamma)}(K+1)$ have property $(B(K))$, which proves Theorem 2, since $2^{K}>2(\log \log x)^{\gamma}$.

ProOF OF $S(0)$. $S(0)$ means that there is a single divisor $d_{1} \mid n_{(0)}=n_{v(x)}$ with property

$(P(0)) \quad \mu\left(d_{1}\right) \neq 0, \quad\left|\log d_{1}-\log d\right| \geq \log 2 \quad$ for all $d \mid n, d \neq d_{1}, \mu(d) \neq 0$.

We set $z_{0}=\frac{1}{2} v(x)$ and write

$$
n=n_{z_{0}}^{*} p_{1}^{\left(z_{0}\right) \alpha_{1}} \cdots p_{r(n)}^{\left(z_{0}\right) \alpha_{r(n)}}
$$


We claim that for all $n \leq x$ except a set of cardinality $\leq x w(x)^{-2 c_{5}(\gamma)}$ the divisor $p_{1}^{\left(z_{0}\right)}$ has property $P(0)$. We denote the exceptional set by $\mathcal{E}(x)$.

$n \in \mathcal{E}(x)$ implies that there is a $d \mid n_{z_{0}}$ such that $\left|\log d-\log p_{1}^{\left(z_{0}\right)}\right|<\log 2$ or that $\left|\log p_{2}^{\left(z_{0}\right)}-\log p_{1}^{\left(z_{0}\right)}\right|<\log 2$. There are $\ll x w(x)^{-A}$ integers $n \leq x$ for which $n_{z_{0}}^{*} \geq x^{1 / 6}$ or $p_{1}^{\left(z_{0}\right)} \geq x^{1 / 6}$ or $\omega\left(n_{z_{0}}^{*}\right) \geq((\log 5) /(\log 2)-1) z_{0}$, by Lemmas 2 and 3 , where $A$ is arbitrarily large. Denote by $m_{z_{0}}^{*}$ any integer equal to $n_{z_{0}}^{*}$ for some $n \leq x$ and by $h(r)$ an integer all of whose prime factors are $>r$.

Then we have

$$
\begin{aligned}
\operatorname{card} \mathcal{E}(x) \ll & \sum_{\substack{m_{z_{0}}^{*}: m_{z_{0}}^{*}<x^{1 / 6} \\
\omega\left(m_{z_{0}}\right)<((\log 5) /(\log 2)-1) z_{0}}} \sum_{p_{1}}^{\prime} \sum_{h\left(p_{1}-1\right) \leq x / m_{z_{0}}^{*} p_{1}} \sum \sum_{\substack{m_{z_{0}}^{*}<x^{1 / 6} \\
p_{1} \geq \exp \exp z_{0}}} \frac{x}{p_{2}: p_{1} \leq p_{2} \leq 2 p_{1}}+x w(x)^{-A} \\
= & \sum_{1}+\sum_{2}+x w(x)^{-A}, \quad \text { say . }
\end{aligned}
$$

In $\sum_{p_{1}}^{\prime}$ the sum is extended over all $p_{1}$ with $\exp \exp z_{0} \leq p_{1}<x^{1 / 6}$ for which there exists a $d \mid m_{z_{0}}$ with $\left|\log d-\log p_{1}\right|<\log 2$.

Since now $m_{z_{0}}^{*} p_{1} \leq x^{1 / 3}$, the last sum $\sum_{h\left(p_{1}-1\right) \leq x / m_{z_{0}}^{*} p_{1}}^{\prime}$ is $\ll x / m_{z_{0}}^{*} p_{1} \log p_{1}$ by the sieve. Thus we obtain

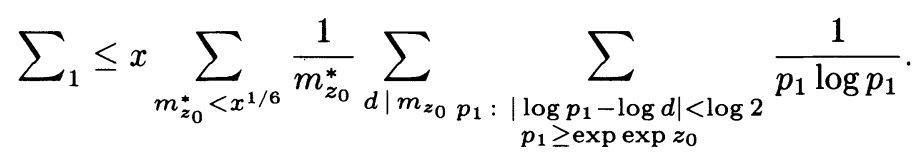

In the inner sum $\log \log p_{1}$ is contained in an interval of length $\ll e^{-z_{0}}$. Thus the $p_{1}$-sum is $\ll e^{-2 z_{0}}$.

We get

$$
\sum_{1} \ll x \sum_{m_{z_{0}}^{*}<x^{1 / 6}} \frac{1}{m_{z_{0}}^{*}} e^{-z_{0}}\left(\frac{5}{2}\right)^{z_{0}} e^{-z_{0}}
$$

By the sieve

$$
\operatorname{card}\left\{n \leq x: n_{z_{0}}^{*}=m_{z_{0}}^{*}\right\} \sim x e^{-z_{0}} / m_{z_{0}}^{*}
$$

such that

$$
\sum_{m_{z_{0}}^{*} \leq x^{1 / 6}} \frac{x}{m_{z_{0}}^{*}} e^{-z_{0}} \ll x
$$

Thus $\sum_{1} \ll x(5 / 2 e)^{z_{0}}$.

For $\sum_{2}$ we get

$$
\sum_{2} \ll \sum_{m_{z_{0}}^{*} \leq x^{1 / 6}} \sum_{p_{1} \geq \exp \exp z_{0}} \frac{x}{m_{z_{0}}^{*} p_{1} \log p_{1}} \ll x e^{-z_{0}} .
$$

This concludes the proof of $S(0)$.

Induction step $S(k) \Rightarrow S(k+1)$. The induction step is similar to the proof of Theorem 2 in $[8]$ but there are additional difficulties. Since the induction step is rather complicated, we start by giving an outline. 
Outline of the induction step. Assume that $n$ has property $(B(k))$ and let the block of $2^{k}$ divisors of $n_{(k)}: d_{1}, \ldots, d_{2^{k}}$ be contained in the interval $I_{k}=\left[\log d_{1}-\right.$ $\left.\zeta_{k}, \log d_{2 k}+\zeta_{k}\right]$. We then consider the translates $I_{k}+\log d$, where $d$ consists of larger prime factors of $n$. Our aim is to show that almost always two such translates merge into a block of the double size $2^{k+1}$.

That would conclude the induction step $S(k) \Rightarrow S(k+1)$ if the $d$ 's are not too large. The aim, to establish the merger of two translates, is roughly achieved as follows:

We denote by $B(k, l)$ the exceptional set of integers for which no two translates $I_{k}+\log d, I_{k}+\log d^{\prime}, d, d^{\prime} \mid n_{(k, l)}$ have merged. We then will show that $\operatorname{card} B(k, l)$ is exponentially decreasing for increasing $l$. We have already shown in Lemma 4 that the measure of

$$
\bigcup_{\substack{d d^{\prime} \mid \tilde{n}_{(k, l)} \\ \mu\left(d d^{\prime}\right)=1}} \log \left(d^{\prime} / d\right)+[-\eta, \eta]
$$

is fairly large for most $\eta$.

This leaves many possibilities for the subsequent prime divisors $p_{1}^{(k, l)}$ and $p_{2}^{(k, l)}$ that the difference $\log p_{2}^{(k, l)}-\log p_{1}^{(k, l)}$ is close to a $\operatorname{logarithm} \log \left(d^{\prime} / d\right)$. But then $\log d+\log p_{1}^{(k, l)}+I_{k}$ contains the block of $2^{k+1}$ divisors:

$$
\log d_{j}+\log d+\log p_{1}^{(k, l)}, \quad \log d_{j}+\log d^{\prime}+\log p_{2}^{(k, l)} \quad\left(j=1, \ldots, 2^{k}\right) .
$$

Thus, if $n_{(k, l)}$ does not have property $(B(k))$ and therefore by definition $n_{(k, l)} \in$ $B(k, l)$, the conditional probability that for small $j, n_{(k, l+j)}$ still does not have property $(B(k))$ and thus $n_{(k, l+j)} \in B(k, l+j)$ is not too close to 1 . This fact accounts for the shrinking of $B(k, l)$ with increasing $l$.

There is one additional difficulty to overcome. We have to guarantee that the new larger block of $2^{k+1}$ divisors is not interrupted by other divisors with different $\mu$-values. This is accomplished by only considering translates $I_{k}+\log d$, which do not contain $\log \tilde{d}$-values other than the translates of the $\log d_{j}$. We will call such divisors $d$ pure.

Thus instead of the measure of

$$
\mathcal{E}(n, k, l, \eta)=\bigcup_{\substack{d d^{\prime} \mid \tilde{n}_{(k, l)} \\ \mu\left(d d^{\prime}\right)=1}} \log \left(d^{\prime} / d\right)+[-\eta, \eta]
$$

we have to consider the measure of

$$
D(n, k, l, \eta)=\bigcup_{\begin{array}{c}
d d^{\prime} \mid \tilde{n}_{(k, l)} \\
\mu\left(d d^{\prime}\right)=1 \\
d, d^{\prime} \text { pure }
\end{array}} \log \left(d^{\prime} / d\right)+[-\eta, \eta] .
$$

In Lemma 5 we will show that the contribution in $\mathcal{E}(n, k, l, \eta)$ of $d, d^{\prime}$ that are not pure is very small. Thus meas $D(n, k, l, \eta)$ is approximately meas $\mathcal{E}(n, k, l, \eta)$.

After this outline we now give the details of the induction step.

DEFINITIONS. We denote by $B(k)$ the set of all $n \leq x$ that possess property $(B(k))$ and by $B(k, l)$ the set of all integers $n \leq x$ that possess the property $(B(k))$, 
but for which there exists no block of $2^{k+1}$ divisors $d_{j}, 1 \leq j \leq 2^{k+1}, d_{j} \mid n_{(k, l)}$ with property $(P(k+1))$.

Given $n \in B(k, l)$ and a block of $2^{k}$ divisors $d_{j} \mid n_{(k)}, 1 \leq j \leq 2^{k}$, with property $(P(k))$. We set $I_{k}\left(n_{(k)}\right)=\left[\log d_{1}-\zeta_{k}, \log d_{2 k}+\zeta_{k}\right]$. If there are several blocks we arbitrarily choose one of them to define $I_{k}\left(n_{k}\right)$. Many of the following definitions will depend on this choice of $I_{k}\left(n_{(k)}\right)$.

Given any positive integer $r$, we call $d \mid n / n_{(k)}^{*} r$-pure if $I_{k}\left(n_{(k)}\right)+\log d$ contains no $\log d^{\prime}, d^{\prime} \mid(n, r)$ other than the translates $\log d^{\prime}:=\log d_{j}+\log d\left(1 \leq j \leq 2^{k}\right)$. For $\eta>0$ we denote by $\lambda(n, k, l, \eta)$ the Lebesgue measure of the set

$$
D(n, k, l, \eta)=\bigcup_{\substack{d d^{\prime} \mid \tilde{n}_{(k, l)} \\ \mu\left(d d^{\prime}\right)=1 \\ d, d^{\prime} n_{(k, l)} \text {-pure }}} \log \left(d^{\prime} / d\right)+[-\eta, \eta] .
$$

Let now $\varepsilon_{2}>0$ be a constant to be determined later. Then we define

$$
L_{k}=\rho^{k}\left(\rho-1-2 \varepsilon_{2}\right) v(x) \quad \text { and } \quad M_{k}=\rho^{k}\left(\rho-1-\varepsilon_{2}\right) v(x) .
$$

We will prove

LEMMA 5. For all $n \in B(k)$ except a set of cardinality $\ll_{\gamma} x \exp \left(-c_{6}(\gamma) w(x)\right)$ we have $\mu(n, k, l, \eta)-\lambda(n, k, l, \eta) \leq \exp \left(r_{k, l}\left(1-c_{7}(\gamma)\right)\right)$, for $L_{k}<l \leq M_{k}$, where $c_{6}(\gamma)>0, c_{7}(\gamma)>0$ depend only on $\gamma$.

In preparation for the proof of Lemma 5 we first give some more definitions and prove some auxiliary lemmas.

We set $q_{k}=r_{k+1}-r_{k}$ and $s_{k}=\left[q_{k}\left(1+\varepsilon_{3}\right)\right]$, where $\varepsilon_{3}>0$ will be determined later.

We denote by $R(k)$ the set of all $n \in B(k)$ with the following properties:

(i) $n_{\left(k, q_{k}\right)} \mid n_{(k)}^{\left(s_{k}\right)}$

(ii) $\omega\left(n_{(k)}\right) \leq r_{k}\left(1+\varepsilon_{4}\right)$,

(iii) $\log p_{s}^{(k)} \geq \exp \left(r_{k}\left(1-\varepsilon_{5}\right)+s\right)$ for $1 \leq s \leq s_{k}$,

(iv) $n_{(k)}^{*\left(s_{k}\right)} \leq x^{1 / 6}$

LEMMA 6. $\operatorname{card}(B(k) \backslash R(k)) \leq C\left(\gamma, \varepsilon_{3}, \varepsilon_{4}, \varepsilon_{5}\right) x \exp \left(-c\left(\gamma, \varepsilon_{3}, \varepsilon_{4}, \varepsilon_{5}\right) v(x)\right)$ where the constants $c>0$ and $C>0$ depend only on the indicated parameters.

ProOF. For any of the properties (i)-(iii) we estimate the set of $n \leq x$ not possessing this property by Lemma 3. For the estimate of the set exceptional with respect to (iv) we observe that $\log \log p_{s_{k}}^{(k)} \leq r_{k}+s_{k}\left(1+\varepsilon_{3}\right)$ for most $n$ and then apply Lemma 2 for the estimate of $n_{\left(k, s_{k}\left(1+\varepsilon_{3}\right)\right)}^{*}$; observing that $k \leq K$ and thus $r_{k} \leq(\log \log x)^{1-\varepsilon_{1}}$.

DEFINITIONS. We introduce the set

$$
\mathcal{F}(n, k, l)=\left\{d \mid \tilde{n}_{(k, l)}: d \text { not } n_{(k, l)} \text {-pure }\right\} .
$$

For $d \mid \tilde{n}_{(k, l)}$ we define $c_{k, l}(d, n)=\operatorname{card}\left\{d^{\prime} \mid \tilde{n}_{(k, l)}:\left(d, d^{\prime}\right)=1\right\}$ and obtain

$$
\mu(n, k, l, \eta)-\lambda(n, k, l, \eta) \leq 2 \eta \sum_{d \in \mathcal{F}(n, k, l)} c_{k, l}(d, n)=2 \eta C(n, k, l), \quad \text { say. }
$$


Thus

$$
\sum_{n \in R(k)}(\mu(n, k, l, \eta)-\lambda(n, k, l, \eta)) \leq 2 \eta \sum_{n \in R(k)} C(n, k, l) .
$$

We introduce the set

$$
\mathcal{G}(n, k)=\left\{d \mid \tilde{n}_{k}^{\left(s_{k}\right)}: d \text { not } n_{k}^{\left(s_{k}\right)} \text {-pure }\right\}
$$

and define

$$
b_{k}(d, n)=\operatorname{card}\left\{d^{\prime} \mid \tilde{n}_{k}^{\left(s_{k}\right)}:\left(d, d^{\prime}\right)=1\right\} .
$$

Since $L_{k}<l \leq M_{k}$, we have for $n \in R(k): \tilde{n}_{(k, l)} \mid \tilde{n}^{\left(s_{k}\right)}$ and therefore $c_{k, l}(d, n) \leq$ $b_{k, l}(d, n)$ and $\mathcal{F}(n, k, l) \subseteq \mathcal{G}(n, k)$. Therefore we have the majorization

$$
C(n, k, l) \leq \sum_{d \in \mathcal{G}(n, k)} b(d, n) .
$$

We introduce the sequence of sets

$$
\nLeftarrow(n, k, s)=\left\{d \mid \tilde{n}_{k}^{(s)}, d \text { not } n_{k}^{(s)} \text {-pure }\right\}, \quad 1 \leq s \leq s_{k},
$$

such that

$$
\mathcal{H}\left(n, k, s_{k}\right) \supseteq \mathcal{G}(n, k) \quad \text { for all } n \in \mathcal{R}(k) \text {. }
$$

We set

$$
B(k, s)=\sum_{n \in \mathcal{R}(k)} \sum_{d \in \mathcal{H}(n, k, s)} b_{k}(d, n)
$$

such that

$$
B\left(k, s_{k}\right) \geq \sum_{n \in \mathbb{R}(k)} C(n, k, l) \quad \text { for } L_{k} \leq l \leq M_{k}
$$

We now prove

LEMMA 7. For $1 \leq s \leq s_{k}, \varepsilon_{6}>0$ we have

$$
B(k, s) \leq C\left(\gamma, \varepsilon_{3}, \varepsilon_{4}, \varepsilon_{5}, \varepsilon_{6}\right) x \exp \left(-r_{k}\left(1-\varepsilon_{5}\right) 2^{r_{k}\left(1+\varepsilon_{4}\right) s_{k}}\left(3 / 2+\varepsilon_{6}\right)^{s} .\right.
$$

PROOF. If $d \in \mathcal{H}(n, k, s)$ we have $d=d^{*}$ or $d=d^{*} p_{s}^{(k)}$, where $d^{*} \mid n_{(k)}^{(s-1)}$. We have

$$
\begin{aligned}
& B(k, s)=\sum_{n \in \mathcal{R}(k)}\left\{\sum_{d^{*} \in \mathcal{H}(n, k, s-1)} b_{k}\left(d^{*}, n\right)+b_{k}\left(d^{*} p_{s}^{(k)}, n\right)\right. \\
& +\sum_{\substack{d^{*} \mid n_{k}^{(s-1)}, d^{*} \notin \mathcal{H}(n, k, s-1) \\
d^{*} p_{s}^{(k)} \in \mathcal{H}(n, k, s)}} b_{k}\left(d^{*} p_{s}^{(k)}, n\right)
\end{aligned}
$$

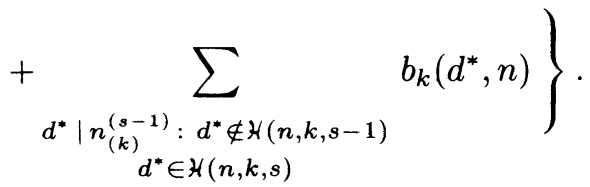


Since $b_{k}\left(d^{*} p_{s}^{(k)}, n\right)=\frac{1}{2} b_{k}\left(d^{*}, n\right)$, we have

$$
\left.\begin{array}{rl}
B(k, s)= & \frac{3}{2} B(k, s-1) \\
& +\sum_{n \in R(k)}\left(\sum_{\substack{d \mid \tilde{n}_{(k)}^{(s-1)}: d \notin \mathcal{H}(n, k, s-1) \\
d p_{s}^{(k)} \in \mathcal{H}(n, k, s)}} b_{k}\left(d p_{s}^{(k)}, n\right)+\sum_{\substack{d \mid \tilde{n}_{(k)}^{(s-1)} \\
d \notin \mathcal{H}(n, k, s-1) \\
d \in \mathcal{H}(n, k, s)}} b_{k}(d, n)\right) \\
= & \frac{3}{2} B(k, s-1)+E(k, s), \quad \text { say . }
\end{array}\right)
$$

Estimate of $E(k, s)$. We have

$$
E(k, s) \leq \sum_{n \in R(k)} \sum_{d \mid \tilde{n}_{(k)}^{(s-1)}}^{\prime} s^{2_{k}-\omega(d)},
$$

where the $\sum^{\prime}$-sum is extended over all $d \mid \tilde{n}_{k}^{(s-1)}$ for which there exists a $\tilde{d} \mid n_{(k)}^{(s-1)}$ with $\log \tilde{d} \in \log \left(d p_{s}^{(k)}\right)+I_{k}\left(n_{(k)}\right)$ or a $\tilde{d} \mid n_{(k)}^{(s-1)}$ with $\log d \in \log \left(\tilde{d} p_{s}^{(k)}\right)+I_{k}\left(n_{(k)}\right)$. Denoting the interval $I_{k}\left(n_{(k)}\right)$ by $\left[a_{k}\left(n_{(k)}\right), b_{k}\left(n_{(k)}\right)\right]$ we have for $s \geq 2$

$$
\begin{gathered}
E(k, s) \ll \sum_{\substack{l \in B(k): l \leq x^{1 / 6} \\
\omega\left(l / l_{(k)}^{*}\right)=s-1, \omega\left(l_{(k)}\right) \leq r_{k}\left(1+\varepsilon_{4}\right) \\
\log p_{s-1}^{(k)}(l) \geq \exp \left(r_{k}\left(1-\varepsilon_{5}\right)+(s-1)\right)}} 1 \\
\cdot \sum_{p}^{\prime \prime} \sum_{l \cdot p \cdot h(p-1) \leq x} 1
\end{gathered}
$$

where the $\sum^{\prime \prime}$-sum is extended over all $p \geq p_{s-1}^{(k)}$ for which

$$
\begin{gathered}
\left|\log p+\log d+a_{k}\left(l_{(k)}\right)-\log \tilde{d}\right|<\log 2 \quad \text { or } \\
\left|\log d+a_{k}\left(l_{(k)}\right)-\log \tilde{d}-\log p\right|<\log 2 .
\end{gathered}
$$

We recall that $h(r)$ denotes an integer all of whose prime factors are $>r$. Since $l \cdot p \leq 2 x^{1 / 3}$ the inner sum is $\ll x /(l p \log p)$ by the sieve.

The interval for $\log \log p$ in $\sum_{p}^{\prime \prime}$ has length $\ll 1 / \log p_{s-1}^{(k)}$ such that

$$
\sum^{\prime \prime} \frac{x}{l p \log p} \ll \frac{x}{l\left(\log p_{(s-1)}^{(k)}\right)^{2}} .
$$

Moreover,

$$
\sum_{\substack{\tilde{d} \mid l \\ \mu(\tilde{d}) \neq 0}} \sum_{\substack{d \mid\left(l / l_{(k)}^{*}\right) \\ \mu(d) \neq 0}} 2^{s_{k}-\omega(d)} \ll 2^{s_{k}-s} 3^{s} 2^{\omega(l)} .
$$


Thus,

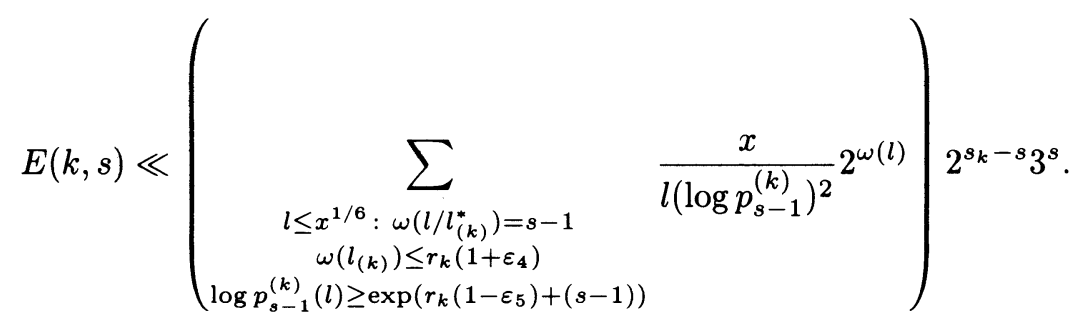

Since

$$
\operatorname{card}\left\{n \leq x: n_{(k)}^{*(s-1)}=l\right\} \gg \frac{x}{l \log p_{s-1}^{(k)}} \quad \text { for } l \leq x^{1 / 6}
$$

we obtain

$$
\sum_{l \leq x^{1 / 6}: \omega\left(l / l_{(k)}^{*}\right)=s-1} \ll x
$$

Therefore,

$$
E(k, s) \ll x 2^{r_{k}\left(1+\varepsilon_{4}\right)+s_{k}} \exp \left(-r_{k}\left(1-\varepsilon_{5}\right)-(s-1)\right) 3^{s}
$$

for $s \geq 2$.

The estimate of $E(k, 1)$ is accomplished in a similar manner. We omit the condition $\log p_{s-1}^{(k)} \geq \exp \left(r_{k}\left(1-\varepsilon_{5}\right)+(s-1)\right)$ and observe instead, that $\log p_{1}^{(k)} \geq$ $\exp r_{k}$. This leads to the estimate (3.6) also for $s=1$.

Now we prove Lemma 7 by induction in $s$. We choose the integer $s_{0}=s_{0}\left(\varepsilon_{6}\right)>0$ such that

$$
\varepsilon_{6}(3 / 2)^{s_{0}} \geq(3 / e)^{s_{0}} .
$$

First it is easily proven by induction, using (3.5) and (3.6), that

$$
B(k, s) \leq x C_{0}(5 / 2)^{s},
$$

where

$$
C_{0}=C^{\prime}\left(\gamma, \varepsilon_{3}, \varepsilon_{4}, \varepsilon_{5}\right) \exp \left(-r_{k}\left(1-\varepsilon_{5}\right)\right) 2^{r_{k}\left(1+\varepsilon_{4}\right)+s_{k}}
$$

for $s \leq s_{0}$. This gives

$$
B(k, s) \leq x C_{0}\left(\frac{5}{3}\right)^{s_{0}}\left(\frac{3}{2}\right)^{s_{0}} \leq x C_{0}\left(\frac{5}{3}\right)^{s_{0}}\left(\frac{3}{2}+\varepsilon_{6}\right)^{s_{0}} .
$$

For $s \geq s_{0}$ we continue the induction, observing (3.5), (3.6), and (3.7). This concludes the proof of Lemma 7.

ProOF OF LemMa 5. From (3.3), (3.4), and Lemma 7 we obtain that

$$
\begin{aligned}
& \sum_{n \in \mathcal{R}(k)}(\mu(n, k, l, \eta)-\lambda(n, k, l, \eta)) \\
& \quad \leq 2 \eta C\left(\gamma, \varepsilon_{3}, \varepsilon_{4}, \varepsilon_{5}, \varepsilon_{6}\right) x \exp \left(r_{k}\left(1-\varepsilon_{5}\right)\right) 2^{r_{k}\left(1+\varepsilon_{4}\right)+s_{k}}\left(\frac{3}{2}+\varepsilon_{6}\right)^{s_{k}} .
\end{aligned}
$$

We now fix the constants $c_{7}(\gamma), \varepsilon_{2}, \ldots, \varepsilon_{6}$ in a manner such that

$$
\left(\rho-1-2 \varepsilon_{2}\right)>(\log 3-1)^{-1}\left(1+\varepsilon_{2}\right)
$$


and

$$
\begin{gathered}
-\left(1-\varepsilon_{5}\right)+\left\{1+\varepsilon_{4}+(\rho-1)\left(1+\varepsilon_{3}\right)\right\} \log 2 \\
+(\rho-1)\left(1+\varepsilon_{3}\right) \log \left(\frac{3}{2}+\varepsilon_{6}\right) \\
\leq\left[1+\left(\rho-1-2 \varepsilon_{2}\right)\left(1-2 c_{7}(\gamma)\right)\right],
\end{gathered}
$$

which is possible because of (3.1) and (3.2). Then (3.8) gives, that

$$
\sum_{n \in \mathcal{R}(k)}(\mu(n, k, l, \eta)-\lambda(n, k, l, \eta)) \ll_{\gamma} \exp \left(r_{k, l}\left(1-2 c_{7}(\gamma)\right)\right)
$$

for $L_{k}<l \leq M_{k}$. This implies that

$$
\mu(n, k, l, \eta)-\lambda(n, k, l, \eta) \leq \exp \left(r_{k, l}\left(1-c_{7}(\gamma)\right)\right)
$$

for all $n \in R(k)$ except a set of cardinality $\ll_{\gamma} x \exp \left(-r_{k, l} c_{7}(\gamma)\right)$. This together with Lemma 6 implies Lemma 5. Because of (3.9) Lemma 4 is applicable. As an immediate corollary of Lemmas 4 and 5 we obtain

LEMMA 8. We have $\lambda(n, k, l, \eta) \geq \exp \left(r_{k, l}\right) w(x)^{-3}$ for all $n \in B(k)$ except a set of cardinality $\ll_{\gamma} x w(x)^{-c_{5}(\gamma)}$.

Conclusion of the Proof of Theorem 2. To complete the induction step and thus the proof of Theorem 2 we want to show that

$$
\operatorname{card} B(k, l) \leq c_{4}(\gamma) x w(x)^{-c_{5}(\gamma)} \quad \text { for some } l \in\left[L_{k}, M_{k}\right] \text {. }
$$

We denote by $C(k, l)$ the subset of $B(k, l)$ of those integers which satisfy the three extra conditions:

(a) $\log n_{(k, l)}^{*} \leq \exp \left(r_{k, l}\right) w(x)$

(b) $\omega\left(n_{(k, l)}\right) \leq 2 r_{k, l}$,

(c) $\lambda(n, k, l, \eta) \geq \exp \left(r_{k, l}\right) w(x)^{-3}$.

By Lemma 2,3 , and 8 we have

$$
\operatorname{card}(B(k, l) / C(k, l)) \ll_{\gamma} x w(x)^{-c_{5}(\gamma)} .
$$

Thus to complete the proof of Theorem 2 it suffices to show that

$$
\operatorname{card} C(k, l) \leq x w(x)^{-2 c_{5}(\gamma)} \quad \text { for some } l \in\left[L_{k}, M_{k}\right]
$$

Assume that

$$
n=n_{(k, l)}^{*} p_{1}^{(k, l)} \cdots p_{r}^{(k, l)}, \quad p_{1}^{(k, l)} \leq \cdots \leq p_{r}^{(k, l)} .
$$

We consider the set $\mathcal{A}(k, l)$ of $n \in \mathcal{C}(k, l)$, whose prime factors $p_{1}^{(k, l)}, p_{2}^{(k, l)}, p_{3}^{(k, l)}$ satisfy the following conditions:

$$
\begin{gathered}
\exp \left(r_{k, l}\right) w(x) \leq \log p_{1}^{(k, l)} \leq 2 \exp \left(r_{k, l}\right) w(x), \\
\log p_{2}^{(k, l)}-\log p_{1}^{(k, l)} \in \bigcup_{\substack{d d^{\prime} \mid \tilde{n}_{(k, l)} \\
d d^{\prime}}}^{n_{(k, l)} \text {-pure }} \underbrace{(k, l)} \geq \log \left(d^{\prime} / d\right)+\left[-\eta_{k+1}, \eta_{k+1}\right] \\
\left.\log p_{3}^{(k, l)} p_{1}^{(k, l)} p_{2}^{(k, l)}\right) .
\end{gathered}
$$

These conditions ensure that there exists a block of $2^{k+1}$ divisors of $n_{(k, l+j)}, j \leq$ $2 \log w(x)$, satisfying $(P(k+1))$, namely the divisors $p_{1}^{(k, l)} d^{\prime} d_{i}, p_{2}^{(k, l)} d d_{i}(1 \leq i \leq$ $2^{k}$ ). Condition (iii) ensures that this block is not destroyed by larger prime factors. 
Thus $C(k, l+j) \subseteq C(k, l) / A(k, l)$ such that

$$
\operatorname{card} C(k, l+j) \leq \operatorname{card} C(k, l)-\operatorname{card} A(k, l) .
$$

We now give a lower bound for card $A(k, l)$. Denote by $m_{(k, l)}^{*}$ an integer equal to $n_{(k, l)}^{*}$ for some $n \in C(k, l)$.

We have

$$
\operatorname{card} A(k, l) \gg \sum_{m_{(k, l)}^{*} p_{1}^{(k, l)} p_{2}^{(k, l)}} \sum_{h\left(m_{(k, l)}^{*} p_{1}^{(k, l)} p_{2}^{(k, l)}\right) \leq x}^{*} 1
$$

where $*$ means that $n_{(k, l)}^{*} \in B(k)$ and that the $n_{(k, l)}^{*}, p_{i}^{(k, l)}$ satisfy (i)-(iii).

By the sieve we have

$$
\operatorname{card} A(k, l) \gg \sum_{m_{(k, l)}^{*}, p_{1}^{(k, l)}, p_{2}^{(k, l)}}^{*} \frac{x}{m_{(k, l)}^{*} p_{1}^{(k, l)} p_{2}^{(k, l)} \log p_{2}^{(k, l)}} .
$$

For a fixed pair $\left(m_{(k, l)}^{*}, p_{1}^{(k, l)}\right)$ the $p_{2}^{(k, l)}$ cover a union of at most $3^{\omega\left(\tilde{m}_{(k, l)}\right)} \leq 3^{2 r_{k, l}}$ disjoint intervals with total logarithmic length $\geq \frac{1}{2} \exp \left(r_{k, l}\right) w(x)^{-3}$. Moreover all the limit points have logarithm of order $\exp \left(r_{k, l}\right) w(x)$. This implies that the $p_{2}^{(k, l)}$ sum is $\gg \exp \left(-r_{k, l}\right) w(x)^{-5}$. The $p_{1}^{(k, l)}$-sum is $\gg 1$. Finally,

$$
\begin{aligned}
\operatorname{card} A(k, l) \gg & \left(\sum_{m_{(k, l)}^{*}: \log m_{(k, l)}^{*} \leq \exp \left(r_{k, l}\right) w(x)} \frac{x}{m_{k, l}^{*}}\right) \exp \left(-r_{k, l}\right) w(x)^{-5}, \\
\operatorname{card} C(k, l) & \leq \sum_{m_{(k, l)}^{*} h\left(\exp \exp r_{k, l} \leq x / m_{(k, l)}^{*}\right.} 1 \\
& \ll\left(\sum_{m_{(k, l)}^{*}: \log m_{(k, l)}^{*} \leq \exp \left(r_{k, l}\right) w(x)} \frac{x}{m_{(k, l)}^{*}}\right) \exp \left(-r_{k, l}\right) .
\end{aligned}
$$

Thus

$$
\operatorname{card} A(k, l) \gg \operatorname{card} C(k, l) w(x)^{-5} \text {. }
$$

Together with (3.11) this gives

$$
\operatorname{card} C\left(k, M_{k}\right) \leq \operatorname{card} C(k, l)\left(1-w(x)^{-5}\right)^{\left(M_{k}-L_{k}\right) / 2 j} \ll x \exp \left(-w(x)^{1 / 2}\right),
$$

which is sufficient.

\section{REFERENCES}

1. P. Erdös and R. R. Hall, On the Möbrius function, J. Reine Angew. Math. 315 (1980), 121-126.

2. P. Erdös and I. Katai, Non complete sums of multiplicative functions, Period. Math. Hungar. 1 (1971), 209-212.

3. P. Erdös and G. Tenenbaum, Sur les diviseurs consécutifs d'un entier, Bull. Soc. Math. France 111 (1983), 125-145.

4. H. Halberstam and H.-E. Richert, On a result of $R$. $R$. Hall, J. Number Theory 11 (1979), 76-89.

5. R. R. Hall, A problem of Endös and Katai, Mathematika 21 (1974), 110-113. 
6. __ Sums of imaginary powers of the divisors of integers, J. London Math. Soc. (2) 9 (1974-75), 571-580.

7. C. Hooley, On a new technique and its applications to the theory of numbers, Proc. London Math. Soc. (3) 38 (1979), 115-151.

8. H. Maier and G. Tenenbaum, On the set of divisors of an integer, Invent. Math. 76 (1984), 121-128.

9. __ On the normal concentration of divisors, J. London Math. Soc. (2) 31 (1985), 393-400.

10. G. Tenenbaum, Sur la probabilité qu'un entier possède un diviseur dans un intervalle donné, Compositio Math. 51 (1984), 243-263.

Department of Mathematics, The University of Georgia, Athens, Georgia 30602 